\title{
Investigation on Non-Standard Scheme Applied to Euler and Third Order Euler for Initial Value Problems
}

\author{
Nurhafizah Moziyana Mohd Yusop \\ Computer Science Department, \\ Faculty of Science and \\ Defense Technology, \\ National Defence \\ University of Malaysia, \\ Kuala Lumpur, Malaysia \\ Mohd Fahmi Mohamad Amran \\ Computer Science Department, \\ Faculty of Science and \\ Defense Technology, \\ National Defence \\ University of Malaysia, \\ Kuala Lumpur, Malaysia
}

\author{
Mohammad Khatim Hasan \\ Faculty of Information \\ Science and Technology, \\ Universiti Kebangsaan Malaysia, \\ Bangi, Malaysia
}

\author{
Muslihah Wook \\ Computer Science Department, \\ Faculty of Science and \\ Defense Technology, \\ National Defence \\ University of Malaysia, \\ Kuala Lumpur, Malaysia
}

\begin{abstract}
There are many benefits to improve methods of Euler for solving the initial value problem (IVPs). Among the good is a simple implementation and low-cost computational. However, there are problems of accuracy and instability in the Euler method. Therefore, the main purpose of this research is to discuss the improvement of the Euler method using Nonstandard Finite Difference (NSFD) scheme in the Initial Value Problems(IVPs). The study is done using a NSFD in the Euler and the Third Order Euler (TOE) method. Combinations of these methods can provide advantages such as easy implementation, low computational cost and better accuracy. Linear and nonlinear IVPs will be tested using SCILAB. About six various problems used to test with various step sizes. The result obtained shows that the NSFD approaching the exact solution.
\end{abstract}

\section{Keywords}

Non-standard Finite Difference Scheme, Initial Value Problem, Euler

\section{INTRODUCTION}

A finite difference scheme for numerical solution of ordinary and partial differential equations is one of the most frequently used. One of the disadvantages is that the standard finite difference method of the qualitative characteristics of the exact solution is usually not transferred to the numerical solution. This could have an impact on the stability characteristics of the standard approach[1]. Besides, in fact, the limit of step size also do not reach the standard methods.

In reality, the non-standard finite difference method is an extension of the standard finite difference method. Nonstandard finite difference scheme (NSFD) was introduced as an alternative method for solving numerous problems in mathematical models engage of algebra, biology and differentiation, and the chaotic system [2]. Mickens introduced Non-standard schemes in 1988 to resolve some of the issues related to numerical instabilities[3]. In general, nonstandard finite difference schemes (NSFD) is deemed general finite-difference schemes by including some of the 'exact' forms[4]. As a result, there are many advantages by using NSFD compared classical techniques. For example NSFD provide an efficient numerical solution from an aspect of the higher efficiency and better accuracy [4].

Euler's method is also called a tangent line method and is the simplest numerical method for solving IVP in ordinary differential equation. This method was developed by Leonhard Euler in 1768 and it is suitable for quick programming, simple implementation and low-cost computational [5]. However, the accuracy factor persuades researcher to use another complex method to replace Euler method. The primary aim of this investigation is to discover a new solution as accurate as possible with the exact solution. This study explores on how powerful nonstandard finite difference methods compared to standard finite difference method. There are six problems involving order and linearity Initial Value Problems(IVPs) using numerical solution.

In this study, we implement the NSFD in the Euler and the Third Order Euler(TOE) method. The combination of Euler and TOE produce a new function of step size which is called as Nonstandard Euler method and Nonstandard Third Order Euler (NSTOE) method. These four methods were implemented by solving the several of IVPs. The numerical solution be shown after testing using SCILAB as a simulation.

\section{NON-STANDARD}

Currently, NSFD plays an imperative role in the invention of reliable numerical methods in various areas in Sciences and Engineering. Since, the standard finite difference method does not accurately solve some sensitive mathematical problems. NSFD tries to solve that problem. When we discuss about NSFD, we mean those which we consider at least one of the non-standard modelling proposed by Mickens. For this investigation, rule\#2 of [6] was implemented.

"Rule 2 : Denominator functions for the discrete derivatives must, in general, be expressed in terms of more complicated functions of the step-sizes than those conventionally used. (These denominator functions, generally, are functions, that 
are related to particular solutions or properties of the general solution to the differential equation)."

\section{Definition 2.1}

Standard Euler method defined by

$y_{n+1}-y_{n}=h f\left(x_{n}, y_{n}\right)$

According to rule \#2 [6], a new step size as a part denominator function is replaced as a new one. In this research [7] and [8] discussed about new step size. The factor noted in this research is linearity function, order of polynomial and value of $\lambda$.

\section{Definition 2.2}

Define the new step size according to Mickens rule, gives the following new finite difference equation for the equation 1.

$\frac{y_{n+1}-y_{n}}{\left(\frac{1-e^{-\lambda h}}{\lambda}\right)}=-\lambda y_{n}$

Equation 2, will use when a function has fixed $\lambda$ and linearity function is linear. Problem 1,2,3 and 6 will use equation 2 in NSFD.

$\frac{y_{n+1}-y_{n}}{\left(\frac{1-e^{-k h}}{k}\right)}=y_{n}\left(1-y_{n}^{k}\right)$

When linearity function is nonlinear and order polynomial more than 1 be influence problem to use equation 3 . In this investigation problem 4 and 5 will use this equation in developing the step size.

\section{EXPERIMENTAL DETAILS}

Using SCILAB 5.4.0 in the experiment is to prolog the effectiveness of Non-standard Euler. SCILAB is a tool for numerical, programming and powerful graphical environment. It is developed for non profit by French government's world prominent "Institut Nationale de Recherche en Informatique et en Automatique - INRIA (National Institute for Informatics and Automation Research)."

SCILAB work similar to numerical operation in the Matlab and other existing numerical/graphics environments. The different thing using SCILAB is open source software. SCILAB can use the execution of a wide range of operating system, such as UNIX, Windows, Linux, etc.[9]. Now, SCILAB 5.4 introduces Scilab desktop which allows the user to save and restore their customized Scilab windows configuration.

\section{NUMERICAL RESULTS}

This section discusses the results of the six various problems and four different step sizes. Three linear and nonlinear initial value problems were tested. Table 1 refers to a set of problem with exact solution in first order differential equations.

Table 1: Set of Problem First Ordinary Differential Equation

\begin{tabular}{|c|c|c|c|c|}
\hline & Equation & Initial Values & Interval of Integration & Source \\
\hline 1. & $\begin{array}{l}y^{\prime}=-0.5 y \\
\text { Exact solution : } y(x)=e^{-0.5 x}\end{array}$ & $y(0)=1$ & $0 \leq \mathrm{x} \leq 20$ & {$[10]$} \\
\hline 2 & $\begin{array}{l}y^{\prime}=y \\
\text { Exact solution : } y(x)=e^{-x}\end{array}$ & $y(0)=1$ & $0 \leq x \leq 20$ & {$[10]$} \\
\hline 3 & $\begin{array}{l}y^{\prime}=30 y \\
\text { Exact solution : } y(x)=e^{-30 x}\end{array}$ & $y(0)=1$ & $0 \leq x \leq 20$ & {$[10]$} \\
\hline 4 & $y^{\prime}=y(1-y)$ & $y(0)=0.5$ & $0 \leq \mathrm{x} \leq 20$ & {$[8]$} \\
\hline 5 & $\begin{array}{l}\text { Exact solution : } y(x)=\overline{(1-0.5) e^{-x}+0.5} \\
y^{\prime}=y\left(1-y^{3}\right)\end{array}$ & $y(0)=0.5$ & $0 \leq x \leq 20$ & {$[8]$} \\
\hline 6. & $\begin{array}{l}\text { Exact solution : } y(x)=\overline{(1-0.5) e^{-x}+0.5} \\
y^{\prime}=25 y(1-y) \\
\text { Exact solution }: y(x)=\overline{(1-0.5) e^{-25 x}+0.5}\end{array}$ & $y(0)=0.5$ & $0 \leq x \leq 20$ & [7] \\
\hline
\end{tabular}

Six various problems are tested by using Euler Method and TOE method. Next, that method which is improved using Non-standard scheme which is called Non-standard Euler and Non-standard Third Order Euler(NSTOE) method. The following Table 2 to Table 7 display the results of the problems. The result shown in the tables are comparisons exact solution and Euler method, Non-standard Euler Method, TOE method and NSTOE method.

Table 2: Result for Problem 1

\begin{tabular}{lcccc}
\hline Method & $\mathbf{h}=\mathbf{0 . 0 2 5}$ & $\mathbf{h}=\mathbf{0 . 1}$ & $\mathbf{h}=\mathbf{0 . 5}$ & $\mathbf{h}=\mathbf{1}$ \\
\hline Euler & $2.80000 \mathrm{E}-06$ & $1.03000 \mathrm{E}-05$ & $3.53000 \mathrm{E}-05$ & $4.44000 \mathrm{E}-05$ \\
Non-standard Euler & 0 & 0 & 0 & 0 \\
TOE & 0 & 0 & $4.00000 \mathrm{E}-07$ & $3.40000 \mathrm{E}-06$ \\
NSTOE & $1.24000 \mathrm{E}-05$ & $1.30000 \mathrm{E}-05$ & $1.18100 \mathrm{E}-04$ & $5.27600 \mathrm{E}-04$ \\
\hline
\end{tabular}


Table 3: Result for Problem 2

\begin{tabular}{lcccc}
\hline Method & $\mathrm{h}=0.025$ & $\mathrm{~h}=0.1$ & $\mathrm{~h}=0.5$ & $\mathrm{~h}=1$ \\
\hline Euler & $4.630 \mathrm{E}-10$ & $1.356 \mathrm{E}-09$ & $2.060 \mathrm{E}-09$ & $2.061 \mathrm{E}-09$ \\
Non-standard Euler & 0 & 0 & 0 & 0 \\
TOE & 0 & $2.000 \mathrm{E}-12$ & $2.980 \mathrm{E}-10$ & $1.774 \mathrm{E}-09$ \\
NSTOE & $5.880 \mathrm{E}-10$ & $3.621 \mathrm{E}-09$ & $2.979 \mathrm{E}-07$ & $1.770 \mathrm{E}-05$ \\
\hline
\end{tabular}

Table 4: Result for Problem 3

\begin{tabular}{lcccc}
\hline Method & $\mathrm{h}=0.025$ & $\mathrm{~h}=0.1$ & $\mathrm{~h}=0.5$ & $\mathrm{~h}=1$ \\
\hline Euler & $2.650 \mathrm{E}-261$ & $1.607 \mathrm{E}+60$ & $7.000 \mathrm{E}+45$ & $1.770 \mathrm{E}+29$ \\
Non-standard Euler & 0 & 0 & 0 & $6.000 \mathrm{E}-263$ \\
TOE & $2.650 \mathrm{E}-261$ & $1.607 \mathrm{E}+60$ & $4.570 \mathrm{E}+106$ & $1.626 \mathrm{E}+72$ \\
NSTOE & $2.190 \mathrm{E}-166$ & 0 & $1.216 \mathrm{E}+59$ & $4.043 \mathrm{E}+42$ \\
\hline
\end{tabular}

Table 5: Result for Problem 4

\begin{tabular}{lcccc}
\hline Method & $\mathrm{h}=0.025$ & $\mathrm{~h}=0.1$ & $\mathrm{~h}=0.5$ & $\mathrm{~h}=1$ \\
\hline Euler & 0 & 0 & 0 & 0 \\
Non-standard Euler & 0 & 0 & 0 & 0 \\
TOE & 0 & 0 & 0 & 0 \\
NSTOE & 0 & 0 & $3.00000 \mathrm{E}-07$ & $1.56000 \mathrm{E}-05$
\end{tabular}

Table 6: Result for Problem 5

\begin{tabular}{lcccc}
\hline Method & $\mathrm{h}=0.025$ & $\mathrm{~h}=0.1$ & $\mathrm{~h}=0.5$ & $\mathrm{~h}=1$ \\
\hline Euler & 0 & 0 & 0 & 0.6031633 \\
Non-standard Euler & 0 & 0 & 0 & 0 \\
TOE & 0 & 0 & 0 & 0.0067794 \\
NSTOE & 0 & 0 & 0 & 0 \\
\hline
\end{tabular}

Table 7: Result for Problem 6

\begin{tabular}{|c|c|c|c|c|}
\hline Method & $\mathrm{h}=0.025$ & $\mathrm{~h}=0.1$ & $\mathrm{~h}=0.5$ & $\mathrm{~h}=1$ \\
\hline Euler & 0 & 0.4640524 & - & - \\
\hline Non-standard Euler & 0 & 0 & 0 & 0 \\
\hline TOE & 0 & 0.0004205 & - & - \\
\hline NSTOE & 0 & 0 & - & - \\
\hline
\end{tabular}

\section{DISCUSSION}

In this study, a solution of each method is compared with the exact solution. About four different step sizes are used for testing in each problem. Those value are $0.025,0.1,0.5$ and 1 . The step size will give impact in the solution using Euler Method. From Table 2 to Table 4, they display linear IVPs while Table 5 to Table 7 shown nonlinear problems. Anyway, factor of $\lambda$ gave some effect for the solution using NSFD.

Table 2 and Table 3 show that Euler Method using NSFD gave the same answer with the exact solution. This method called Non-standard Euler. The error for each step size gave zero values. While in Table 3, Non-standard Euler gave the same result with exact solution on values of the step size at $0.025,0.1$ and 0.5 . Excluding the value of step size is 1, Nonstandard Euler gave the answer approaching to exact solution rather for another method.
Table 5 to Table 7 display the nonlinear problems. Factor of $\lambda$ and order polynomial give us indication to use the suitable formula. According to Definition 2.2, there are only two ways NSFD scheme used for this investigation. The results also showed that Non-standard Euler gave us the same answer with the exact solution.

Refer to Table 5, all method shown the same answer with exact solution except NSTOE when step sizes are 0.5 and 1 . Since value $\lambda$ and order polynomial is 1 , Equation 2 and Equation 3 gave the same answer with the exact solution. Table 6 discuss that Equation 3 is more suitable for solving Problem 5. This is because the order polynomial gave effect for the solution. When NSFD scheme is used on Euler method and TOE, it gave the same answer with the exact solution. Finally, Table 7 also confirm that Non-standard Euler gave 
the same answer with the exact solution in which Equation 2 is used in Problem 6 where $\lambda=25$.

We can conclude that all problems except problem 5, gave answers that Non-standard Euler is better than NSTOE. Compare to first order method, Third Order Euler method are depending on point. Thus the cumulative rounding error was produced bigger than Third Order Euler truncation error.

\section{CONCLUSION}

The non-standard schemes have been tested numerically in terms of their consistency with known behavior of analytic solution. Non-standard is applied to Euler Method and TOE to give better solutions. The factors of linearity function, order polynomial and value of $\lambda$ play an important role in produce new finite difference equation. New finite difference equation's denominator called as new step size when it is applied to NSFD scheme. The results are presented in the tables which provide comparison of the exact solution with Euler method, Non-standard Euler Method, TOE Method and NSTOE Method. The tables definitely show that non-standard scheme approaching the exact solution, although using huge step sizes.

\section{REFERENCES}

[1] K. Moaddy et al., A New Hybrid Nonstandard Finite Difference Adomian Scheme for Solution of Nonlinear Equations, Sains Malaysiana, 2011, pp. 515-519.

[2] R. E. Mickens, Advances in the Application of Nonstandard Finite Differences Schemes, Singapore : World Scientific, 2005

[3] R. E. Mickens, An Introduction Finite Difference Models of Differential Equations, Journal of Computational Acoustics, 1999, pp 39-59.

[4] P. M. Manning and G. F. Margrave, Introduction to Nonstandard Finite-difference Modeling, CREWES Research Report, 2006, pp 1-10.

[5] S. Fadugba, et al., Euler Method for Solving Initial Value Problems in Ordinary Differential Equations, The Pacific Journal of Science and Technology, 2012, pp 152-158.

[6] R. E. Mickens, Nonstandard Finite Difference Models of Differential Equations, World Scientific, 1994, pp 81-85.

[7] P. Kama, "Non-standard Finite Difference Methods in Dynamical Systems", Ph.D. Thesis, University of Pretoria, 2009.

[8] A. K. Gucoglu, "The Solution of Some Differential Equations by Nonstandard Finite Difference Method", Master Thesis, Izmir Institute of Technology, 2005.

[9] G. E. Urroz, Introduction to SCILAB, 2001, pp 1-17.

[10] Z. B. Ibrahim et al., Block Method for Generalised Multistep Adams and Backward Differentiation Formulae in Solving First Order ODEs, MATEMATIKA, 2005, pp 25-33 